\title{
Obtención y caracterización del polvo de bronce Cu88Sn6,5Zn4Pb1,5 para
}

\section{aplicaciones en cojinetes $(\cdot)$}

\author{
Natalia Krivij*, Xenia Suárez*, José Villalonga* y Alejandro Cores ${ }^{* *}$ \\ Resumen El trabajo tiene como objetivo el desarrollo del polvo de bronce aleado \\ Cu88Sn6,5Zn4Pb1,5 para sustituir el material originalmente utilizado en la fabricación de \\ cojinetes con propiedades antifricción. Se realizó la caracterización física y química del \\ polvo, y se empleó un diseño experimental $2^{3}$ con vistas a determinar los parámetros \\ óptimos del proceso tecnológico de sinterización del polvo, para el caso específico de la \\ fabricación del cojinete de sellaje del subconjunto eje-sello del compresor abierto de \\ refrigeración.
}

Palabras clave Polvo de bronce aleado. Atomización. Pulvimetalurgia.

\section{Obtaining and characterizing Cu88Sn6,5Zn4Pb1,5 bronze powder for bearing applications}

\begin{abstract}
The aim of this work is the development of alloyed bronze powder Cu88Sn6,5Zn4Pb1,5 to substitute the material used in the manufacture of bearings with antifriction properties. The physical and chemical characterization of the powder has been carried out and an experimental $2^{3}$ design to determine the optimal parameters of the technological process of powder sintering has been used in the specific case of sealed bearing manufacture of the subset shaft-seal of the open cooling compressor.
\end{abstract}

Keywords

Alloyed bronze powder. Spraying molten metal. Powder metallurgy.

\section{INTRODUCCIÓN}

Dentro de la gama de piezas producidas por el Departamento de Pulvimetalurgia del Centro de Investigaciones Metalúrgicas (CIME), el mayor volumen lo ocupan las piezas antifricción (cojinetes), empleándose en su producción, fundamentalmente, polvo de bronce al estaño (Sn $10 \%$ ) con o sin adición de grafito. Los cojinetes obtenidos tienen buena calidad y aceptación. No obstante, debido al alto costo y difícil adquisición del estaño se ha venido trabajando en el desarrollo de otros polvos metálicos aleados con otros elementos, que permitan disminuir el contenido de estaño y mantener las propiedades requeridas para los materiales antifricción ${ }^{[1]}$.

Son conocidos los bronces aleados con la presencia de plomo y zinc, denominados bronces rojos; estos son bronces cuaternarios $(\mathrm{Cu}, \mathrm{Sn}, \mathrm{Zn}, \mathrm{Pb})$ que tienen una elevada aceptación y empleo. La presencia de zinc en estas aleaciones mejora sus propiedades, aumentando la resistencia, dureza, mecaniza- ción, plasticidad y resistencia a la corrosión. El contenido de zinc fluctúa entre 2 y $8 \%$, aumentando el contenido a medida que disminuye el de estaño. El plomo mejora la mecanización y la resistencia al desgaste y disminuye el coeficiente de fricción, además mejora la compactación de los polvos ${ }^{[2]}$.

Como resultado de las buenas propiedades que se obtienen con este tipo de bronce, se desarrolla y caracteriza el polvo de bronce Cu88Sn6,5Zn4Pb1,5, que puede ser muy utilizado en la fabricación de diferentes tipos de casquillos y cojinetes. A partir de este material, se realiza la producción a escala experimental del cojinete de sellaje (Fig. 1) del subconjunto eje-sello del compresor abierto de refrigeración ${ }^{[3]}$.

\section{PLANTEAMIENTO DEL TRABAJO}

El objetivo fundamental es realizar el estudio del polvo de bronce aleado, con el fin de utilizarlo en diversas aplicaciones para la elaboración de

(•) Trabajo recibido el día 27 de abril de 2000 y aceptado en su forma final el día 30 de noviembre de 2000.

$\left(^{*}\right)$ Centro de Investigaciones Metalúrgicas (CIME). Avda. 51, 23611, La Lisa, Ciudad de La Habana (Cuba).

$\left(^{* *}\right)$ Centro Nacional de Investigaciones Metalúrgicas (CENIM). Avda. Gregorio del Amo, 8. 28040 Madrid (España). 


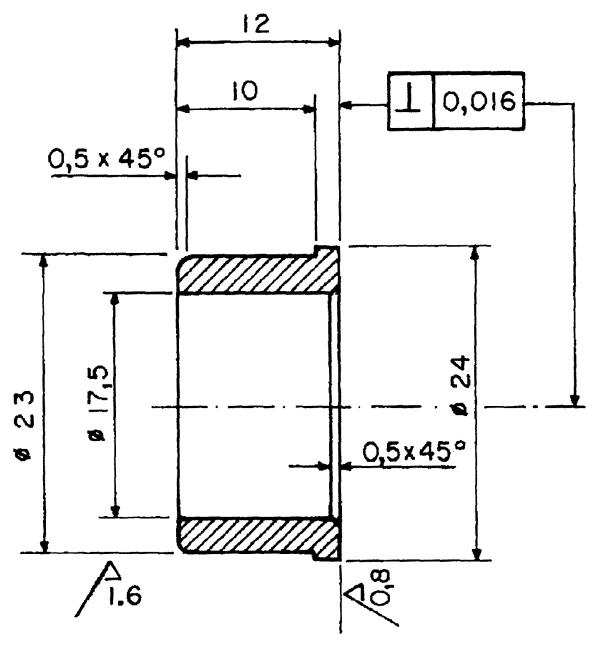

Figura 1. Cojinete de sellaje.

Figure 1. Seal boss.

cojinetes, tomando como partida el caso específico de la elaboración del cojinete de sellaje del compresor abierto de refrigeración.

El compresor abierto de refrigeración se produce con la calidad necesaria para ser empleado en unidades condensadoras de baja, media y alta temperatura, operando con refrigerante freón 12. Estas unidades se utilizan en gabinetes helados, congeladores y refrigeradores comerciales, así como en cámaras de refrigeración, vitrinas de exhibición, instalaciones de climatización, etc.

En el conjunto eje-sello del compresor, la pieza debe poseer:

- Desviaciones y tolerancias de forma y posición adecuadas.

- Rugosidad superficial y acabados precisos en la superficie de contacto.

- Características adecuadas de dureza y resistencia.

A través de los métodos de fabricación de piezas por pulvimetalurgia y al obtener bronce aleado con buenas propiedades físico- químicas, tecnológicas y mecánicas, deben cumplirse satisfactoriamente los requisitos técnicos necesarios.

\section{PARTE EXPERIMENTAL}

\subsection{Obtención de polvo Cu88Sn6,5Zn4Pb1,5}

El polvo de bronce aleado se obtiene por atomización de la aleación fundida con agua a alta presión, siguiendo el esquema que se muestra en la figura $2^{[3]}$.

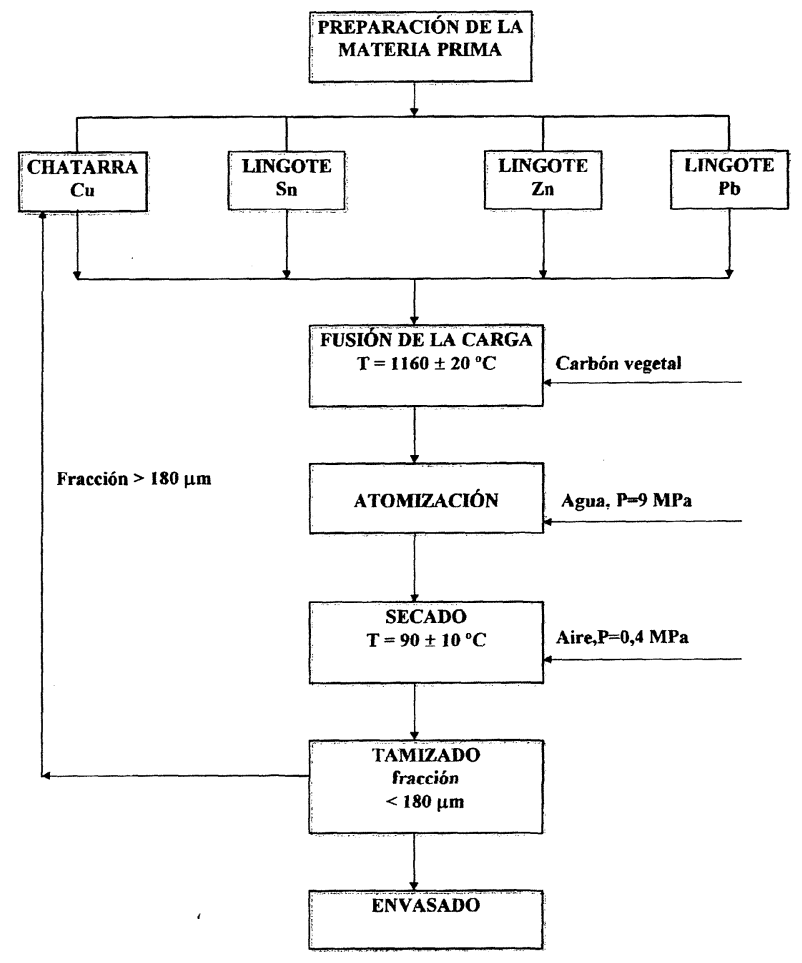

Figura 2. Esquema tecnológico de la producción de polvo de bronce aleado.

Figure 2. Technological scheme for alloyed bronze powder production.

En la tabla I se presentan los componentes para fundir $100 \mathrm{~kg}$ de aleación, teniendo en cuenta las pérdidas de los metales que componen la aleación. La instalación de atomización del CIME se incluye en la figura 3.

El crisol del horno cargado con el cobre se calienta hasta $1.160^{\circ} \mathrm{C}$; posteriormente se introduce el zinc, homogeneizando bien el baño metálico; luego, se introducen conjuntamente el estaño y el plomo. La aleación se calienta hasta 1.160 $\pm 20^{\circ} \mathrm{C}$ y la temperatura debe ser controlada mediante un termopar de inmersión. Se retira la escoria y el carbón vegetal que queda en la superficie y, después de lograda una buena homogeneidad en

Tabla I. Composición de la carga para fundir $100 \mathrm{~kg}$ de aleación

Table I. Charge composition to melt $100 \mathrm{~kg}$ of alloy

\begin{tabular}{cccc}
\hline & & \multicolumn{2}{c}{ Pérdida durante la fusión } \\
\cline { 3 - 4 } Metal & Carga, kg & \multicolumn{1}{c}{$\mathbf{k g}$} & $\%$ \\
\hline $\mathrm{Cu}$ & 88,88 & 0,88 & 1,0 \\
$\mathrm{Sn}$ & 6,60 & 0,098 & 1,5 \\
$\mathrm{Zn}$ & 4,08 & 0,08 & 2,0 \\
$\mathrm{~Pb}$ & 1,58 & 0,015 & 1,0 \\
\hline
\end{tabular}

http://revistademetalurgia.revistas.csit: $\mathbf{E} 3$ 


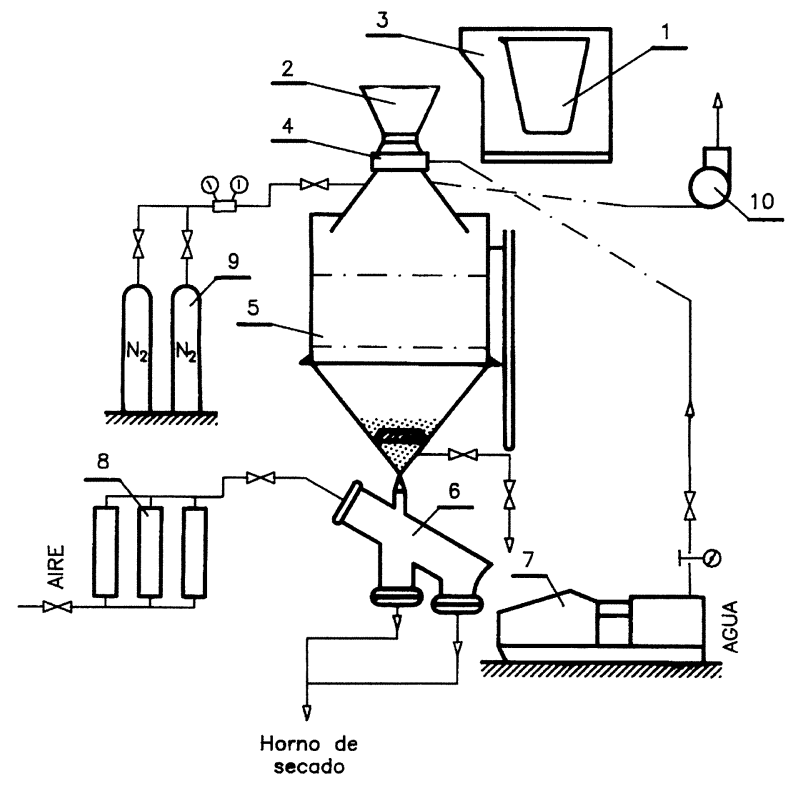

Figura 3. Esquema de la instalación de pulverización con agua a alta presión: 1) Crisol de grafito, 2) Colector de metal líquido, 3) Horno de inducción, 4) Inyector para la atomización, 5) Cámara de atomización, 6) Colector de polvos, 7) Bomba de émbolo, 8) Calentador de aire, 9) Batería de botellas de oxígeno, 10) Extractor.

Figure 3. Scheme of powdering plant with water at high pressure: 1) Graphite melting pot, 2) Collector liquid metal, 3) Induction furnace, 4) Powdering injector, 5) Powdering chamber, 6) Powder collector, 7) Piston pump, 8) Blast heather, 9) Battery nitrogen gas, 10) Extractor

toda la aleación fundida, ésta queda lista para ser atomizada.

La característica más importante de los polvos es el tamaño de las partículas. Son varios los factores que influyen en la calidad de los polvos metálicos obtenidos mediante la atomización. Según Titov et al. ${ }^{[4]}$ los tamaños de partículas más finas son favorecidos por la baja viscosidad del metal, la baja tensión superficial de éste, el pequeño diámetro de la tobera de salida del metal, la alta presión de atomización, el gran volumen de agente pulverizador y el ángulo óptimo de ataque del chorro de metal, entre otras.

La tecnología de inyección del metal en el molde usando alta presión para la atomización del agua, ha experimentado un desarrollo reciente ${ }^{[5]}$.

El efecto del tamaño del grano sobre el comportamiento mecánico de los materiales nanocristalinos ha sido evaluado usando el método de los elementos finitos ${ }^{[6 \mathrm{y} 7]}$. Al suponer que el material es plástico elástico perfecto, se ha demostrado que la tensión aumenta y el módulo elástico disminuye con una disminución en el tamaño del grano.

Se ha estudiado el polvo de aleaciones de cobre de 2 a $10 \mathrm{~nm}$, producido por atomización con agua y consolidando la aleación mediante presión isostática a alta temperatura ${ }^{[8 \text { y } 9]}$.

En el Departamento de Pulvimetalurgia del CI$\mathrm{ME}$, mediante la experiencia acumulada en la fabricación de polvos metálicos obtenidos por atomización con agua ${ }^{[10-12]}$, se ha llegado a la conclusión de que para obtener altos rendimientos de la fracción menor de $180 \mu \mathrm{m}$ de polvo de bronce, el diámetro de la tobera de salida del chorro de metal debe estar entre 9 y $10 \mathrm{~mm}$, ya que con la utilización de diámetros inferiores existe el riesgo de que se enfríe y obstruya la salida de metal líquido. La temperatura de metal se recomienda que sea de $150^{\circ} \mathrm{C}$ por encima de su punto de fusión y la presión de atomización debe ser de 90-100 atm.

Esta experiencia se utiliza para atomizar el polvo de bronce aleado Cu88Sn6,5Zn4Pb1,5. En la cámara de atomización se produce una mezcla de polvo y agua que permanece en reposo 15-20 min. En este tiempo, el polvo se sedimenta en el colector de polvos de la cámara de atomización. En el colector el polvo se deshidrata, extrayendo el agua de la mezcla con aire a presión. El polvo, aún húmedo, que se extrae del colector, se seca en una estufa a una temperatura de $90 \pm 10^{\circ} \mathrm{C}$.

Durante el tamizado se separan las fracciones de polvo que se utilizan en la producción de piezas $(<180 \mu \mathrm{m})$, las que no son útiles se reciclan como parte de la carga inicial del proceso de fusión.

El polvo tamizado se somete a un recocido con el objetivo de eliminar el endurecimiento de las partículas y la reducción de los óxidos presentes, a una temperatura de 0,4-0,6 la temperatura de fusión del metal.

\subsection{Propiedades del polvo}

La caracterización del polvo de bronce Cu88Sn6,5Zn4Pb1,5 se realizó mediante una serie de ensayos que permiten evaluar las propiedades físico-químicas, tecnológicas y mecánicas.

\subsubsection{Análisis químico}

El análisis químico se llevó a cabo por el método de absorción atómica. En la tabla II se ofrecen los resultados.

\subsubsection{Análisis granulométrico}

En la tabla III se ofrece el análisis granulométrico determinado según la Norma ISO 4497. Como se puede apreciar, la granulometría del polvo 
Tabla II. Análisis químico del polvo, \% en masa

Table II. Chemical analysis of the powder, \% in mass

\begin{tabular}{cccc}
\hline $\mathrm{Cu}$ & $\mathrm{Sn}$ & $\mathrm{Zn}$ & $\mathrm{Pb}$ \\
88,3 & 6,0 & 4,2 & 1,5 \\
\hline
\end{tabular}

Tabla III. Análisis granulométrico del polvo, \% en masa

Table III. Granulometric analysis of the powder, \% in mass

\begin{tabular}{cr}
\hline Fracción, $\mathrm{mm}$ & $\%$ \\
\hline$>0,177$ & 0,84 \\
$0,177-0,150$ & 2,11 \\
$0,150-0,105$ & 11,30 \\
$0,105-0,088$ & 4,14 \\
$0,088-0,037$ & 46,86 \\
$<0,037$ & 34,78 \\
\hline
\end{tabular}

obtenido es menor de $0,180 \mathrm{~mm}$ según estaba previsto de acuerdo a los parámetros de atomización seleccionados para este tipo de aleación (diámetro de la tobera de salida del chorro de metal de 9-10 $\mathrm{mm}$, a la temperatura del metal, $150^{\circ} \mathrm{C}$ por encima de su punto de fusión, y a la presión de atomización de 90-100 atm). Esta composición granulométrica es típica del proceso de obtención del polvo por los métodos de atomización.

\subsubsection{Análisis microestructural}

El análisis microestructural se realizó en un microscopio óptico-metalográfico, "Xarl Zeiss" modelo Neophot 30. Se tomo una foto sin ataque químico con aumento a 100 y dos fotos con ataque a 100 y 250 de aumento.

En la figura 4 se aprecia una porosidad homogénea muy bien distribuida en toda la estructura. El plomo se observa alojado en los espacios interpartículas. La estructura revela solución sólida $\alpha$ de $\mathrm{Sn}$ en $\mathrm{Cu}$ en forma de granos maclados muy dispersos, como se aprecia en las figuras 5 y 6 .

\subsubsection{Fluidez}

El valor de la fluidez es de $35 \mathrm{~s} / 50 \mathrm{~g}$, según el ensayo realizado utilizando la Norma ISO 4490.

\subsubsection{Densidad aparente}

La densidad aparente es de $3,76 \mathrm{~g} / \mathrm{cm}^{3}$, según el ensayo realizado utilizando las Normas ISO 3923, ISO 3923/2 e ISO 3953.

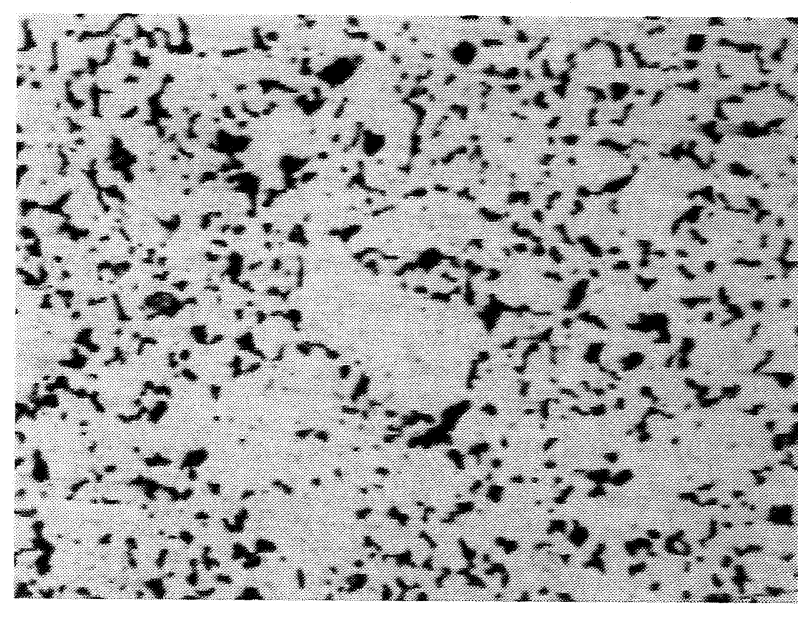

Figura 4. Microestructura del bronce aleado sin ataque (x 100).

Figure 4. Microstructure of alloyed bronze unetched (x 100).

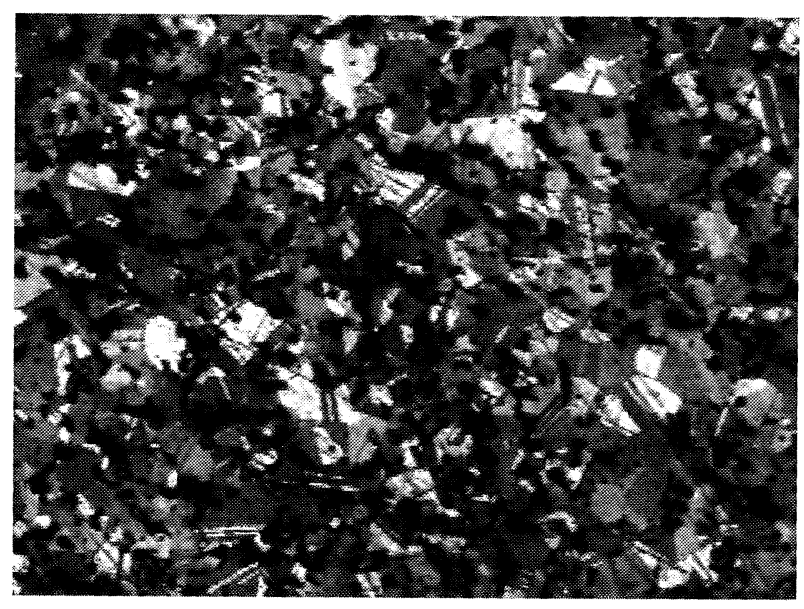

Figura 5. Microestructura del bronce aleado con ataque (x 100).

Figure 5. Microstructure of alloyed bronze etched (x 100).

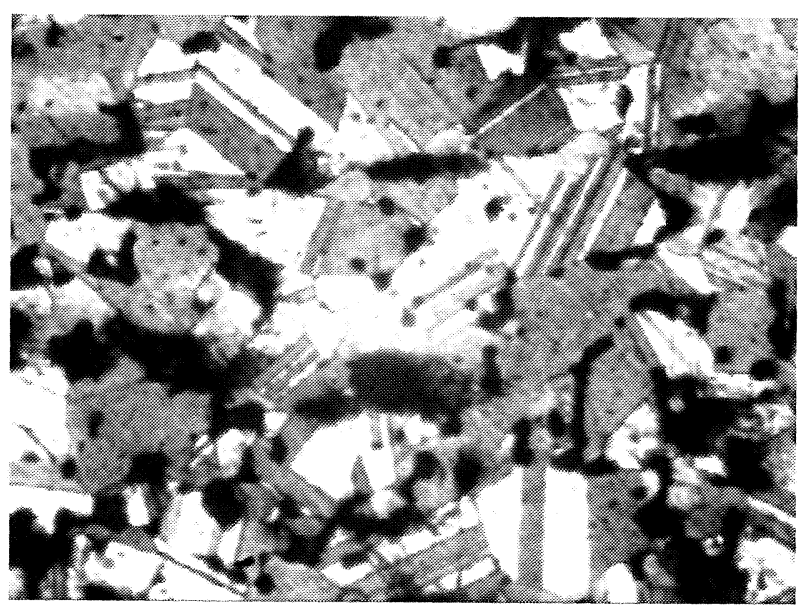

Figura 6. Microestructura del bronce aleado con ataque (x 250).

Figure 6. Microstructure of alloyed bronze etched (x 250). 


\subsubsection{Compactación}

En un estudio reciente sobre el proceso de compactación $^{[13]}$ se ofrece una nueva ecuación para la curva de densidad-presión de compactación, que facilita la caracterización del comportamiento a la consolidación del polvo mediante dos parámetros:

a) Medida de la capacidad del compacto para aumentar su densidad por deformación plástica.

b) Densidad del polvo sin aplicación de presión.

Los modelos de simulación para representar la compactación de los polvos son capaces de predecir la distribución de las densidades y fijar los niveles de fuerza en la compactación ${ }^{[14 \text { y 15] }}$. El empleo de estos modelos ofrece unos resultados sorprendentemente razonables.

El ensayo de compactación se ha realizado según la Norma ISO 3927 para analizar la variación, inversamente proporcional, de la densidad y la porosidad, en la medida que aumenta la presión ejercida sobre las probetas en verde, como se evidencia en la tabla IV.

\subsection{Diseño experimental}

La determinación los parámetros óptimos de elaboración de las piezas con las propiedades mecánicas requeridas ${ }^{\left[11^{1} 12\right]}$, a partir de polvo de bronce aleado obtenido por atomización, se efectuó mediante un diseño experimental de $2^{3}$, tomándose como variables el rendimiento a la dureza aparente y la resistencia a la compresión radial.

Variable Nivel bajo (-) Nivel alto (+)

$X_{1}$ - Presión de $\quad 400 \quad 600$

compactación, $\mathrm{MPa}$

$\mathrm{X}_{2}$ - Temperatura de $740 \quad 780$

sinterización, ${ }^{\circ} \mathrm{C}$

$\mathrm{X}_{3}$ - Tiempo de $\quad 30 \quad 90$

sinterización, min

Estos parámetros, la matriz experimental, así como los resultados de dureza y resistencia al comportamiento radial obtenidos para cada tratamiento se reflejan en la tabla V. Cada resultado es el producto de tres mediciones efectuadas.

De esta manera queda el polinomio de la forma sigụiente:

$$
\begin{aligned}
y & =a_{0}+a_{1} X_{1}+a_{2} X_{2}+a_{3} X_{3}+a_{1,1} X_{1}^{2}+a_{2,2} X_{2}^{2} \\
& +a_{3,3} X_{3}^{2}+a_{1,2} X_{1} X_{2}+a_{1,3} X_{1} X_{3}+a_{2,3} X_{2} X_{3}
\end{aligned}
$$

donde,

$y \quad$ es la variable dependiente

$\mathrm{X}_{1}, \mathrm{X}_{2}$ y $\mathrm{X}_{3} \quad$ las variables independientes consideradas

$\mathrm{X}_{1}, \mathrm{X}_{2} \ldots, \mathrm{X}_{1} \mathrm{X}_{3}$ las interacciones dobles de las variables independientes. En cada caso $a_{i l}$ es el factor de influencia de cada variable.

Tabla IV. Ensayo de compactación

Table IV. Compacting assay

\begin{tabular}{ccccccccc}
\hline No & $\begin{array}{c}\text { Presión } \\
\text { MPa }\end{array}$ & $\begin{array}{c}\text { Diámetro } \\
\mathbf{m m}\end{array}$ & $\begin{array}{c}\text { Altura } \\
\mathbf{m}\end{array}$ & $\begin{array}{c}\text { Masa } \\
\mathbf{g}\end{array}$ & $\begin{array}{c}\text { Volumen } \\
\mathbf{c m}^{\mathbf{3}}\end{array}$ & $\begin{array}{c}\text { Densidad } \\
\text { en verde } \\
\mathbf{g} / \mathbf{c m}^{\mathbf{3}}\end{array}$ & $\begin{array}{c}\text { Relación* } \\
\text { Dv/Dc }\end{array}$ & $\begin{array}{c}\text { Porosidad } \\
\text { \% }\end{array}$ \\
\hline 1 & 200 & 11,29 & 12,19 & 7,9 & 1,220 & 6,5 & 0,74 & 26 \\
2 & 200 & 11,29 & 12,00 & 7,9 & 1,201 & 6,6 & 0,76 & 24 \\
3 & 200 & 11,28 & 12,02 & 7,9 & 1,201 & 6,6 & 0,76 & 24 \\
4 & 400 & 11,28 & 11,87 & 8,0 & 1,186 & 6,7 & 0,77 & 23 \\
5 & 400 & 11,28 & 11,95 & 8,0 & 1,194 & 6,7 & 0,77 & 23 \\
6 & 400 & 11,28 & 11,97 & 8,0 & 1,196 & 6,7 & 0,77 & 23 \\
7 & 500 & 11,28 & 11,60 & 7,9 & 1,159 & 6,8 & 0,78 & 22 \\
8 & 500 & 11,28 & 11,58 & 7,9 & 1,157 & 6,8 & 0,78 & 22 \\
9 & 500 & 11,27 & 11,62 & 7,9 & 1,159 & 6,8 & 0,78 & 22 \\
10 & 600 & 11,28 & 11,57 & 8,0 & 1,150 & 7,0 & 0,80 & 20 \\
11 & 600 & 11,28 & 11,50 & 8,0 & 1,149 & 7,0 & 0,80 & 20 \\
12 & 600 & 11,28 & 11,52 & 7,9 & 1,151 & 6,9 & 0,79 & 21 \\
13 & 800 & 11,28 & 10,70 & 7,9 & 1,069 & 7,4 & 0,85 & 15 \\
15 & 800 & 11,28 & 10,21 & 8,0 & 1,020 & 7,8 & 0,89 & 11 \\
\hline
\end{tabular}

* Dv = densidad en verde; $\quad D c=$ densidad compacto 
Tabla V. Características del diseño

Table V. Desing characteristics

\begin{tabular}{|c|c|c|c|c|c|c|c|}
\hline \multirow{2}{*}{$\begin{array}{l}\text { Régimen de } \\
\text { sinterización }\end{array}$} & \multirow[t]{2}{*}{ Tratamiento } & \multirow{2}{*}{$\begin{array}{c}\text { Presión de } \\
\text { compactación } \\
\text { MPa }\end{array}$} & \multirow{2}{*}{$\begin{array}{c}\text { Temperatura } \\
\text { de sinterización } \\
{ }^{\circ} \mathrm{C}\end{array}$} & \multirow{2}{*}{$\begin{array}{c}\text { Tiempo de } \\
\text { sinterización } \\
\text { min. }\end{array}$} & \multicolumn{3}{|c|}{ Matriz de diseño } \\
\hline & & & & & $x_{1}$ & $x_{2}$ & $x_{3}$ \\
\hline \multirow[t]{2}{*}{1} & 1 & 400 & 740 & 30 & - & - & - \\
\hline & 2 & 600 & 740 & 30 & + & - & - \\
\hline \multirow[t]{2}{*}{ II } & 3 & 400 & 780 & 30 & - & + & - \\
\hline & 4 & 600 & 780 & 30 & + & + & - \\
\hline \multirow[t]{2}{*}{ III } & 5 & 400 & 740 & 90 & - & - & + \\
\hline & 6 & 600 & 740 & 90 & + & - & - \\
\hline \multirow[t]{2}{*}{ IV } & 7 & 400 & 780 & 90 & - & + & + \\
\hline & 8 & 600 & 780 & 90 & + & + & + \\
\hline
\end{tabular}

\subsection{Propiedades mecánicas}

\subsubsection{Dureza aparente}

Los mayores valores de dureza ( $32 \mathrm{HB}$ ) se obtienen para las condiciones de ensayos del tratamiento 6 (tabla V), el cual consiste en aplicar una presión de compactación de $600 \mathrm{MPa}$, una temperatura de sinterización de $740^{\circ} \mathrm{C}$ y un tiempo de retención a la temperatura de sinterización de 90 minutos.

Los resultados obtenidos fueron procesados estadísticamente obteniéndose la ecuación de regresión siguiente:

$$
y=52.300+6579 X_{1}-2,064 X_{3}+2,857 X_{1}^{2}
$$

Se puede apreciar que en los niveles estudiados no hubo una influencia significativa de la temperatura de sinterización, el tiempo de retención a esa temperatura influyó negativamente y por el contrario la presión de compactación influyó significativamente de manera positiva.

\subsubsection{Resistencia a la compresión radial}

Los mayores valores de resistencia a la compresión radial se obtienen utilizando el tratamiento 8 (tabla $\mathrm{V}$ ), que consiste en realizar la sinterización a $780^{\circ} \mathrm{C}$ durante $90 \mathrm{~min}$ con probetas prensadas a $600 \mathrm{MPa}$, lográndose una resistencia a la compresión radial de $864 \mathrm{MPa}$.

La ecuación de regresión obtenida es la siguiente:

$$
\begin{gathered}
y=7671,7+4,26 X_{1}-16,51 X_{2}-154,99 X_{3}- \\
100,57 X_{1}^{2}+1,28 X_{3}^{2}+281 X_{1} X_{2}+2,34 X_{1} X_{3}
\end{gathered}
$$

Según se puede apreciar, en los niveles estudiados, influye positivamente sobre la presión de compactación, no siendo así la temperatura de sinterización y el tiempo de retención a esa temperatura, los cuales influyen significativamente y de manera negativa.

\section{ANÁLISIS DE LOS RESULTADOS}

El análisis del diseño experimental efectuado para las dos variantes dependientes, sus ecuaciones de regresión, los valores obtenidos del factor de influencia de cada variable independiente, así como de sus interacciones, nos permite determinar el movimiento de los parámetros hacia el óptimo, previamente considerado, teniendo en cuenta las exigencias técnicas del cojinete del compresor abierto de refrigeración. Para ello se selecciona un posible intervalo de trabajo, dentro del cual debe encontrarse un valor óptimo de dureza y de resistencia a la compresión radial, teniendo en cuenta las condiciones de explotación del cojinete en cuestión.

Para valores restringidos de dureza entre 40 y $70 \mathrm{HB}$ se obtiene un óptimo de $69 \mathrm{HB}$ para una presión de compactación de $600 \mathrm{MPa}$, una temperatura de sinterización de $760^{\circ} \mathrm{C}$ y un tiempo de retención a la temperatura de sinterización de 90 $\min$.

Para valores restringidos de resistencia a la compresión radial entre 300 y $700 \mathrm{MPa}$ se obtiene un óptimo de $672 \mathrm{MPa}$ para una presión de compactación de $600 \mathrm{MPa}, 760{ }^{\circ} \mathrm{C}$ de temperatura de sinterización y 90 min de tiempo de retención a esa temperatura.

Teniendo en cuenta la situación de compromiso que se debe establecer entre la dureza y la resistencia a la compresión radial, y conociendo, 
además, las condiciones de explotación a que va a estar sometida la pieza, la cual requiere alta dureza, no siendo así con la resistencia a la compresión radial debido a que no va a estar sometida a condiciones severas de trabajo, se decide establecer un régimen óptimo que presupone una dureza de 69 $\mathrm{HB}$ y una resistencia a la compresión radial de 672 $\mathrm{MPa}$.

Por tanto, los valores óptimos de los parámetros que influyen en estas propiedades mecánicas son:

Presión de prensado

Temperatura de sinterización

$600 \mathrm{MPa}$ $760^{\circ} \mathrm{C}$

Tiempo de retención a la temperatura de sinterización

$90 \min$

\section{CONCLUSIONES}

En la obtención del bronce aleado para la fabricación de cojinetes con características antifriccionantes, y en particular del cojinete del compresor abierto de refrigeración, se determinaron las características físico-químicas, tecnológicas y mecánicas del polvo Cu88Sn6,5Zn4Pb1,5 obtenido por atomización del metal fundido y se realizaron diferentes ensayos para determinar, entre otros, la compactación y los parámetros tecnológicos óptimos, cuyos valores son: presión de prensado, 600 $\mathrm{MPa}$; temperatura de sinterización, $760^{\circ} \mathrm{C}$ y tiempo de retención durante la sinterización, $90 \mathrm{~min}$. Se obtiene una dureza de $69 \mathrm{HB}$ y una resistencia a la compresión radial de $672 \mathrm{MPa}$.

Se realizó la serie 0 y la producción en serie de esta pieza, a escala de planta piloto, con resultados satisfactorios en las pruebas de explotación en la industria, por lo que se propone la utilización de este material en otras aplicaciones de cojinetes antifricción.

\section{REFERENCIAS}

1. C.C. ERmakov, Poroshkovie Stali y Isdelia. Ed. Machinostroenie, Leningrado, Rusia, 1990, pp. 319.

2. S.S. KipARisov y G. A. Libenson, Metalurgia de los polvos, Ed. Metallurgiya, Moscú, Rusia, 1980, pp. 433-445.

3. W. SuWARDjO y P. SERRANO, Estudio de la obtención de polvo aleado de $\mathrm{Cu}-\mathrm{Sn}-\mathrm{Zn}-\mathrm{Pb}$ con destino a la fabricación de cojinetes antifricción. Proyecto de Investigación, CIME, Ciudad de La Habana, Cuba, 1995.

4. D.N. Titov y A.Y. Stepanov, Tecnología del proceso de fundición, Ed. MIR, Moscú, Rusia, 1981, pp. 461.

5. Y. Tanaka y K. NaKabayashi, Powder Metall. 41 (1998) 47-51.

6. M. B. Bus. Mat. Sci. Eng. A., 161 (1993) 127-132

7. H.S. Kim, C. Suryanarayama, S.J. Kim y B.S. ChUn, Powder Metall. 41 (1998) 217-220.

8. M.J. Tenwick y H.A. Davies, Mat. Sci. Tech. 98 (1988) 543-546.

9. , J.M. SAInz-Borda, I. GutiérRez, F. CASTRO-Fernández, M. Fuentes, H.A. Davies Y C.M. Sellars, Powder Metall. 38 (1995) 31-38.

10. N. Krivij, W. SuWArdjo y A. Cores, Rev. Metal. Madrid 31 (1995) 394-399.

11. N. Krivij, W. Suwardjo, J. Frades, T. Chacón, A. Cores y L. García, Rev. Metal. Madrid 32 (1996) 391. 396.

12. N. Krivij, W. Suwardjo, L. García, A. Cores y A. Formoso, Rev. Metal. Madrid 33 (1997) 229-238.

13. R. Panelli y F. Ambrosio Filho, Powder Metall. 41 (1998) 131-133.

14. J. Kergadallan, G. Puente, P. Doremus y E. Pavier, Proc. Int. Workshop on "Modelling of metal powder forming processes"., Grenoble, Francia (1997) 277-285.

15. P.M. Modnet Computer Modelling Group, Powder Metall. 42 (1999) 301-311. 